\title{
Healthy dietary patterns from food diaries and FFQ are not associated with colorectal cancer risk: results from the UKWCS
}

\author{
Petra Jones ${ }^{1,2}$, Janet Cade ${ }^{2}$, Charlotte Evans ${ }^{2}$, Neil Hancock ${ }^{2}$ and Darren Greenwood ${ }^{2}$ \\ ${ }^{1}$ University of Malta, Msida, Malta and \\ ${ }^{2}$ University of Leeds, Leeds, United Kingdom
}

\section{Abstract}

Dietary pattern analyses have most commonly used food frequency questionnaire (FFQ) data for large population studies, whilst food diaries (FD) tend to be used with smaller datasets and followed up for shorter terms, restricting the possibility of a direct comparison. Studies comparing dietary patterns derived from two different assessment methods, in relation to diet and disease are limited. The aims of this study are to assess the agreement between dietary patterns derived from FFQ and FDs and to compare the associations between the Mediterranean dietary pattern and the World Cancer Research Fund/American Institute of Cancer Research (WCRF/ AICR) dietary pattern in relation to colorectal cancer incidence.

The study population included 2276 healthy middle-aged women - participants of the UK Women's Cohort Study. Energy and nutrient intakes, derived from 4-day FDs and from a 217-item FFQ were compared. A 10 and an 8-component score indicating adherence to the Mediterranean diet and to the 2007 WCRF/AICR cancer prevention recommendations respectively were generated. Agreement was assessed by weighted Kappa statistics and the Bland-Altman method. Cox regression was used to estimate hazard ratios (HRs) for colorectal cancer risk for both the FD and the FFQ patterns, for each score separately.

The Bland-Altman method showed that the FFQ gave a higher energy intake compared to the FD with a bias of -525 kcal (95\% CI $-556,-493)$ between the two methods. Agreement was slight for the Mediterranean diet score (K=0.15; 95\% CI: $0.14,0.16)$ and fair for the WCRF/AICR score ( $\mathrm{K}=0.38 ; 95 \%$ CI: $0.37,0.39)$. A total of 173 incident cases of colorectal cancer were documented. In the multi-variable adjusted models, the estimates for an association with colorectal cancer were weak: HR $=0.94$ (95\% CI: 0.83 to 1.06 ) for a 1-unit increment in the Mediterranean diet score using FD and HR $=1.01(95 \%$ CI: 0.83 to 1.24) for a 1-unit increment in the WCRF/AICR score using FD. For scores derived from the FFQ, estimates were inverse, but weak (HR $=0.80$ (95\% CI: 0.90 to 1.00 ) for a 1-unit increment in the Mediterranean diet score using FFQ and $\mathrm{HR}=0.84$ (95\% CI: 0.67 to 1.05$)$ for a 1-unit increment in the WCRF/AICR score using FFQ.

There is insufficient evidence of an association of colorectal cancer risk with the Mediterranean dietary pattern or with the WCRF/ AICR cancer prevention recommendations, irrespective of the dietary assessment method in this sample. Further studies with larger sample sizes, using FD for diet assessment are warranted.

\section{Conflict of Interest}

There is no conflict of interest. 